EPJ Web of Conferences 49, 14002 (2013)

DOI: $10.1051 /$ epjconf/20134914002

(C) Owned by the authors, published by EDP Sciences, 2013

\title{
W mass and Triple Gauge Couplings at Tevatron
}

\author{
Pierre Pétroff ${ }^{1, a}$ \\ ${ }^{1}$ Laboratoire de l'Accélérateur Linéaire B.P. 34 Orsay 91898 France
}

\begin{abstract}
The W mass is a crucial parameter in the Standard Model (SM) of particle physics, providing constraints on the mass of the Higgs boson as well as on new physics models via quantum loop corrections. On the other hand, any deviation of the triple gauge boson couplings (TGC) from their values predicted by the SM would be also an indication for new physics. We present recent measurements on $W$ boson mass and searches for anomalous TGC (aTGC) in $W \gamma, Z \gamma, W W, W Z$ and $Z Z$ at Fermilab Tevatron both by CDF and D $\varnothing$ Collaborations. The CDF Collaboration has measured the $W$ boson mass using data corresponding to $2.2 \mathrm{fb}^{-1}$ of integrated luminosity. The measurement, performed using electron and muon decays of $W$ boson, yields a mass of $M_{W}=80387 \pm 19 \mathrm{MeV}$. The $\mathrm{D} \emptyset$ Collaboration has measured $M_{W}=80367 \pm 26 \mathrm{MeV}$ with data corresponding to $4.3 \mathrm{fb}^{-1}$ of integrated luminosity in the channel $W \rightarrow e v$. The combination with an earlier D $\varnothing$ result, using independant data sample at $1 \mathrm{fb}^{-1}$ of integrated luminosity, yields $M_{W}=80375 \pm 23 \mathrm{MeV}$. The limits on anomalous TGCs parameters are consistent with the SM expectations.
\end{abstract}

\section{$1 \mathrm{~W}$ mass measurement at the Tevatron}

\subsection{Analysis Strategy}

At the Tevatron, $W$ bosons are primarily produced in $q \bar{q}$ annihilation, $q \bar{q} \rightarrow W+X$, where $X$ can include QCD radiation that results in measurable hadronic recoil in events. $W \rightarrow l v_{l}$ decays, where $l=e$ or $\mu$, are selected with high purity by the $\mathrm{CDF}$ and $\mathrm{D} \varnothing$ ( $l=e$ only) detectors and used to measure the mass of the $W$ boson.

$M_{W}$ is determined using three kinematic variables measured in the plane perpendicular to the beam direction: the lepton $\left(p_{T}^{e, \mu}\right)$ and neutrino $\left(p_{T}^{v}\right)$ transverse momentum distributions and the transverse mass $m_{T}=$ $\sqrt{2 p_{T}^{e, \mu} p_{T}^{v}(1-\cos \Delta \phi)}$, where $\Delta \phi$ is the opening angle between the lepton and neutrino momenta. The magnitude and direction of $p_{T}^{v}$ is inferred from the missing transverse energy $\left(\vec{E}_{T}\right)$.

A parametrized Monte Carlo simulation (FAST MC) is used to predict the shape of the transverse mass distribution as well as the lepton and neutrino $p_{T}$ distributions as a function of $M_{W}$. The $W$ boson mass is extracted by fitting the $M_{W}$ prediction of the Monte Carlo simulation to the data with a binned maximum-likelihood fit.

The line-shapes prediction depend on the kinematic distributions of the $W$ decay products and detector effects, which are constrained from control samples and theoretical calculations. The kinematic distributions are determined by several effects inluding internal QED radiation, the intrinsic $W$ boson transverse momentum, and the proton parton distribution functions (PDFs). Electron efficiency, hadronic recoil modelling, calorimeter

\footnotetext{
a e-mail: petroff@lal.in2p3.fr
}

response both to electromagnetic shower and hadronic shower and calorimeter fiducial acceptance are among the most important detector effects for both experiments. The parametrized fast simulation enables a study of these effects at a level below 1 part in $10^{4}$.

\subsection{Event Generation and Simulation}

Kinematics are simulated using RESBOS [1] which is a next-to-leading order generator including non-perturbative effects at low boson $p_{T}$ [4]. The PDFs used in RESBOS, are CTEQ6.6 [3]. They affect the $W$ boson mass measurement through their effects on the kinematics of decay charged leptons. The dominant effect from QED radiation to the $W$ boson mass measurement comes from radiation of photons from the final state charged lepton. These processes are simulated by combining the PHOTOS program [2] with RESBOS.

The techniques used by CDF and D $\varnothing$ Collaborations to determine the single most important ingredient, namely the lepton energy scale are very different. The CDF measurement exploits precise measurements of track momenta in the inner tracker, while the $\mathrm{D} \varnothing$ mesurement relies on a detailed understanding of electron showers in the calorimeter.

At CDF, the high statistics $J / \Psi$ and $\Upsilon(1 S) \rightarrow \mu \mu$ quarkonia, are used to set the momentum scale. Fig 1 (left) shows the measurement of the $Z$-boson mass in the dimuon channel. The $Z$ boson mass measured is $M_{Z}=91180 \pm 12_{\text {stat }}$ $\pm 10_{\text {syst }} \mathrm{MeV}$, in good agreement with the world average $(91188 \pm 2 \mathrm{MeV})$.

The electromagnetic calorimeter energy scale is set using the peak of the $E / p$ electron distribution from $W \rightarrow e v$ 

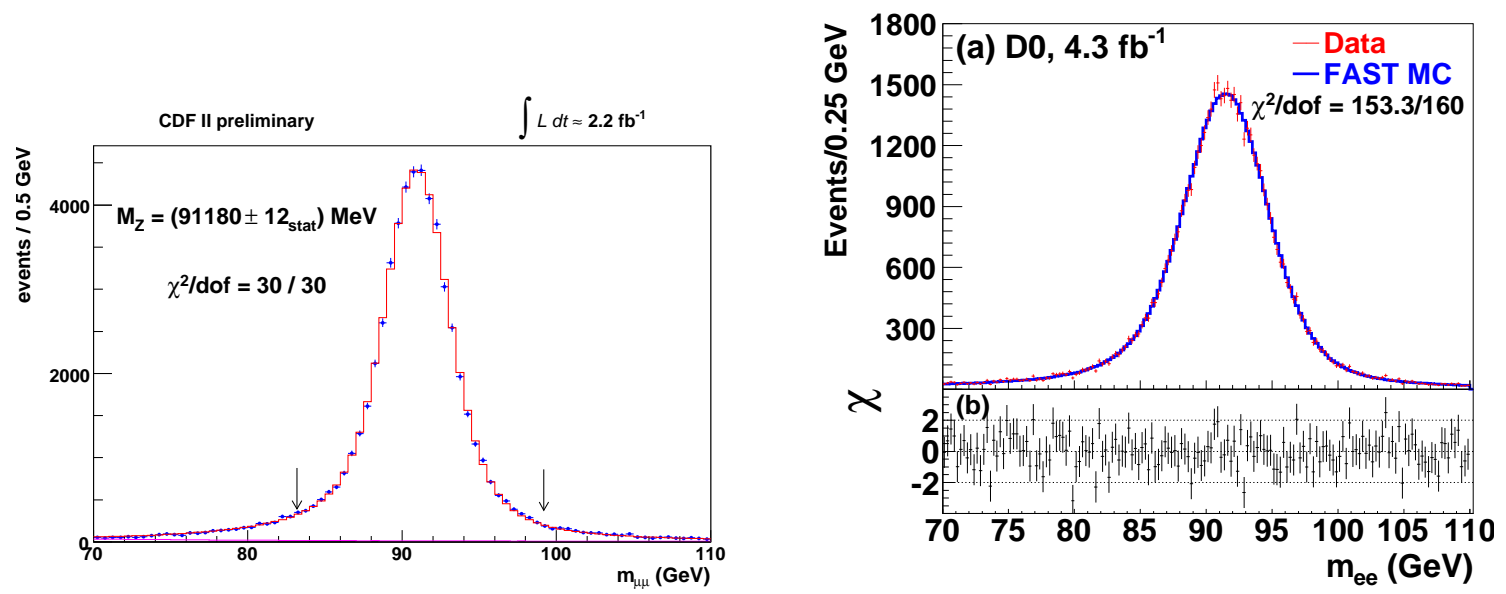

Figure 1. CDF (left): The $Z \rightarrow \mu \mu$ mass fit, showing the data (points) and the best fit simulation template (histogram). The arrows indicate the fitting range. $\mathrm{D} \emptyset$ (right):(a) Distribution of the di-eelctron invariant mass in $Z \rightarrow e^{+} e^{-}$sample. The shape of the distribution of the FAST MC simulation, normalised to the data, is superimposed. (b) Bin-by-bin difference between data and prediction, divided by the statistical uncertainty in the data.

and $Z \rightarrow e e$ samples. The $Z \rightarrow e e$ mass is fitted to crosscheck the energy scale and the non-linearity. A value of $M_{Z}=91230 \pm 30_{\text {stat }} \pm 14_{\text {syst }} \mathrm{MeV}$ is obtained, consistent with the world average. The tail of the $E / p$ distribution is used to tune the absolute number of radiation lengths in the tracker material.

At D $\varnothing$ the calorimeter energy scale is calibrated using $Z \rightarrow$ $e^{+} e^{-}$. The corrections for energy lost in uninstrumented regions are based on a comparison between data and detailed GEANT-based simulation of the D $\varnothing$ detector. The precise mesurements of the $Z$ boson mass from LEP [7], is used to determine the absolute energy scale of the calorimeter. The $M_{W}$ measurement presented here is effectively a measurement of the ratio of $W$ and $Z$ boson masses. Figure 1 (right) shows a comparison of the $Z$ boson mass distributions for data and fast simulation.

The hadronic energy in the event contains the hadronic system recoiling from the $W$ boson, the effects of low energy products from spectator parton collisions and other beam collisions, final state radiation, and energy from the recoil particles that enter the electron selection window. The hadronic response and resolution is calibrated using the mean and width of the $\eta_{i m b}$ distribution in $Z \rightarrow e e$ (and $\mu \mu$ for CDF) events in bins of $p_{T}$ of the leptons. Here, $\eta_{i m b}$ is defined as the projections of the sum of dielectron (dimuon) transverse momentum and the vector sum of the transverse components of the energies measured in the calorimeter cells excluding those associated with the reconstructed electron.

\subsection{Results}

Kinematic distributions of background events passing the event selection cuts are included in the templates fits with their estimated normalizations. The backgrounds in the $\mathrm{W}$ boson samples are $Z \rightarrow e e(\mu \mu)$ in which one $e(\mu)$ escapes detection, multijet in which a jet is misidentified as an electron with $\left(\vec{E}_{T}\right)$ arising from misreconstruction, and
Table 1. CDF and DØ : Uncertainties

\begin{tabular}{lll}
\hline Uncertainty $(\mathrm{MeV})$ & CDF & DØ \\
\hline lepton energy scale and resolution & 7 & 17 \\
Hadronic recoil energy scale and resolution & 5 & 6 \\
Backrounds & 3 & 2 \\
\hline Parton distributions & 10 & 11 \\
QED radiation & 4 & 7 \\
$p_{T}(W)$ model & 5 & 2 \\
\hline Total systematic uncertainty & 15 & 22 \\
$W$ boson statistics & 12 & 13 \\
\hline Total uncertainty & 19 & 26 \\
\hline
\end{tabular}

$W \rightarrow \tau v \rightarrow e(\mu) v v v$. CDF has also evaluated pion and kaon decays in flight and cosmic ray backgrounds for the $\mu$ decay channel. Background fractions is about $7 \%$ for muons(CDF) and $2 \%$ for electrons (CDF and DØ).

Concerning $\mathrm{D} \varnothing$ the final $M_{W}$ uncertainty as well as most of the other uncertainties, are driven by the limited $Z$ statistics (see Table 1). The final $M_{W}$ uncertainty in CDF relies on precise measurements of track momenta in the inner tracker based on the huge $J / \Psi$ and $\Upsilon(1 S)$ statistics (see Table 1). The largest source of uncertainty in the model of $W$ production and decay is the finite precision of the PDFs for both experiments.

The $\mathrm{W}$ boson mass is measured by performing a binned maximum-likehood fit to the lepton $p_{T}^{l}$, neutrino $p_{T}$ and $m_{T}$ distributions for each lepton channel (electron channel only for DØ ). The result of the fits to the $m_{T}$ kinematic distribution is shown in Fig. 2 both for CDF (right) and DØ (left).

By combining all six fits and taking into account correlations [8] CDF has published [5] the following result:

$M_{W}=80387 \pm 12_{\text {stat }} \pm 15_{\text {syst }}=80387 \pm 19 \mathrm{MeV}$.

$\mathrm{D} \varnothing$ is using $m_{T}$ and electron $p_{T}^{e}$ in the combination only as the result from $\vec{E}_{T}$ does not have any significant weight in the combination. The $\mathrm{D} \varnothing$ combination yields to: 

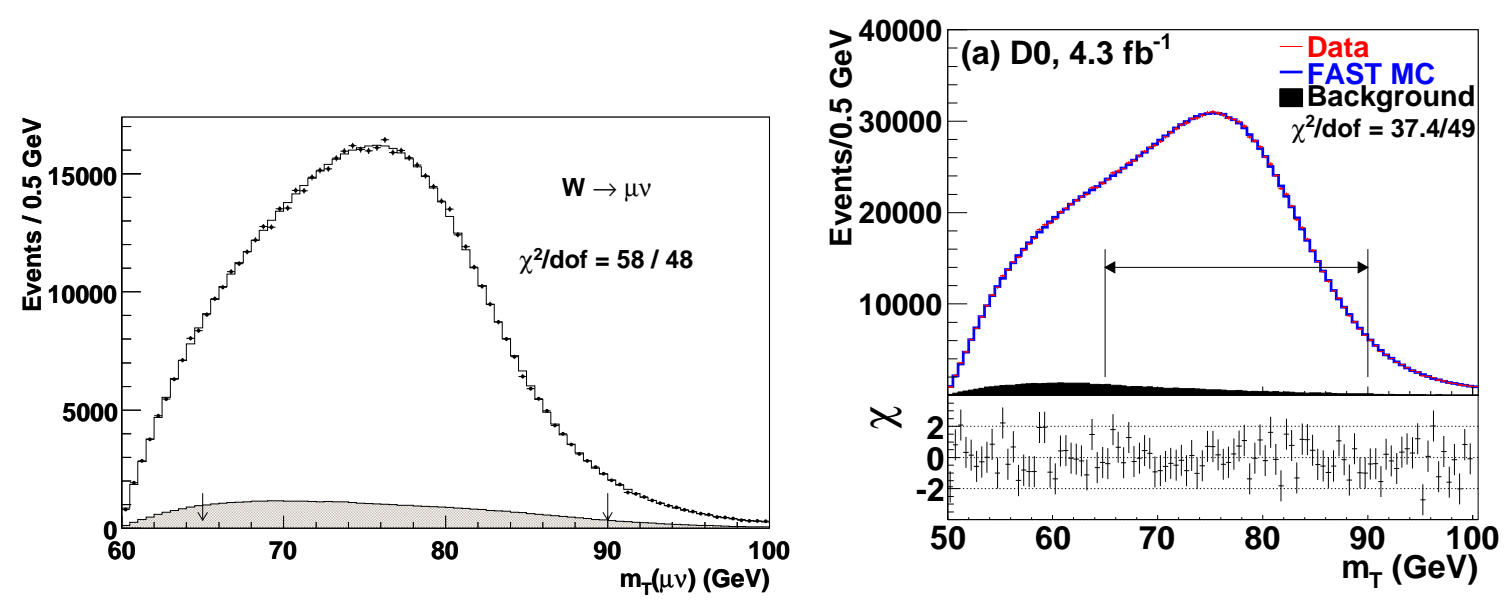

Figure 2. The $W$ transverse mass fit for muon channel in CDF (left). The $W$ transverse mass fit for electron channel in DØ (right). The background contributions to the template are overlaid and arrows indicate the fitting range for both distributions

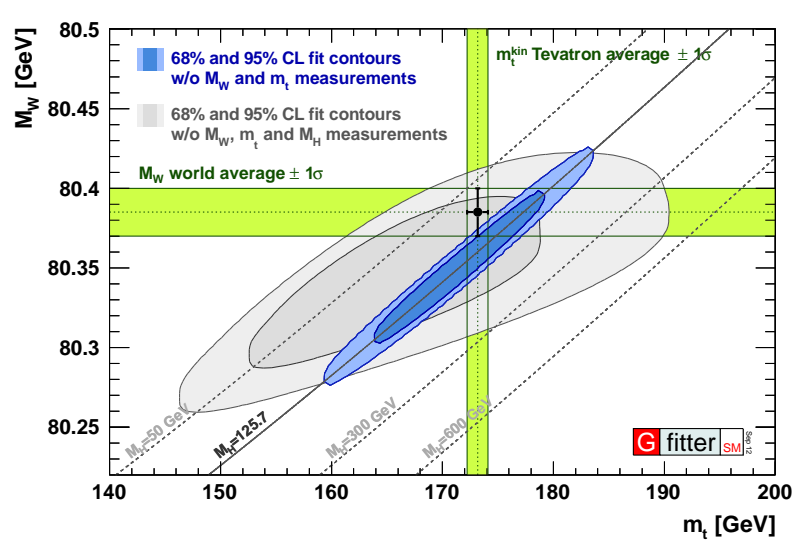

Figure 3. Indirect prediction for the masses of the top quark and the $W$ boson from internal consistency of the SM. This prediction uses the direct measurement of the candidate Higgs boson discovered at the LHC recently. The direct measurements of the $W$ boson mass (the world average dicussed in Sec 1.3) and the Tevatron average of direct measurements of the top quark mass[12] are also shown.

$M_{W}=80367 \pm 13_{\text {stat }} \pm 22_{\text {syst }}=80367 \pm 26 \mathrm{MeV}$.

$\mathrm{D} \varnothing$ further combines this result [6] with the earlier measurement [9] to obtain the new DØ Run II results: $M_{W}=80375 \pm 11_{\text {stat }} \pm 20_{\text {syst }}=80375 \pm 23 \mathrm{MeV}$. The results of the two experiments are of comparable precision, and the measurements are in good agreement. All results from the Tevatron are combined, using again the methods from Ref.[8], to yield the new Tevatron average [10]: $M_{W}=80387 \pm 16 \mathrm{MeV}$. Combining Tevatron and LEP results [7] yields the new world average: $80385 \pm 15$ $\mathrm{MeV}$.

\subsection{Testing the Standard Model (SM)}

Electroweak fit [11] is a powerful tool for the comparison of direct and indirect constraints on the Higgs boson mass.
Moreover, assuming the boson recently discovered at LHC with a mass of $126 \mathrm{GeV}$ is the Higgs boson, electroweak fits can test the validity of the SM. This is shown in Fig. 3 which shows the indirect prediction for the masses of the top quark and the $W$ boson from internal consistency of the SM. This predicton uses the direct measurements of the mass of the candidate Higgs boson. From [11] the indirect constraints prefer a Higgs boson that is consistent within the new boson discovered at LHC at the $1.2 \sigma$ level, with the mass of the new boson. More precise measurements of $M_{W}$ are therefore needed to tighten the indirect constraints and make the test of SM even more stringent.

\section{Triple Gauge Couplings at Tevatron}

\subsection{Methods}

Measurements of the associated production of two vector bosons $(\gamma, W, Z)$ are important tests of the electroweak sector of the SM. The production of a diboson final state can occur by particle-antipaticle annihilation (t-channel) or by boson self-interaction (s-channel). Observing triple gauge couplings (TGCs), not permitted in the SM or with different intensity with respect to the SM predictions, would be a sign of new physics.

In the presence of new physics, observables such as production cross-sections, TGCs, and various kinematic distributions, are expected to deviate from their SM predictions. The charged TGCs present in $W^{+} W^{-}, W Z$, and $W \gamma$ production are $\Delta g_{1}^{V}, \Delta \kappa_{V}$ and $\lambda_{V}(\mathrm{~V}=Z$ or $\gamma)$ where $\Delta$ represents the deviation from SM predictions. In the SM, $\Delta g_{1}^{V}=\Delta \kappa_{V}=\lambda_{V}=0$. Using constraints due to gauge invariance, $\kappa_{Z}$ and $\lambda_{Z}$ can be expressed as a function of the other parameters and the weak mixing angle $\theta_{W}$ as: $\kappa_{Z}=g_{Z}^{1}-\left(\kappa_{\gamma}-1\right) \tan ^{2}\left(\theta_{W}\right)$.

The neutral TGCs $h_{i}^{V}(\mathrm{i}=3,4)$, studied in $Z \gamma$ production, are not allowed in the SM tree-level, and their values are predicted to be zero.

The $p_{T}$ or $E_{T}$ distributions of fully reconstructed candidate bosons or of the charged leptons from boson decays 


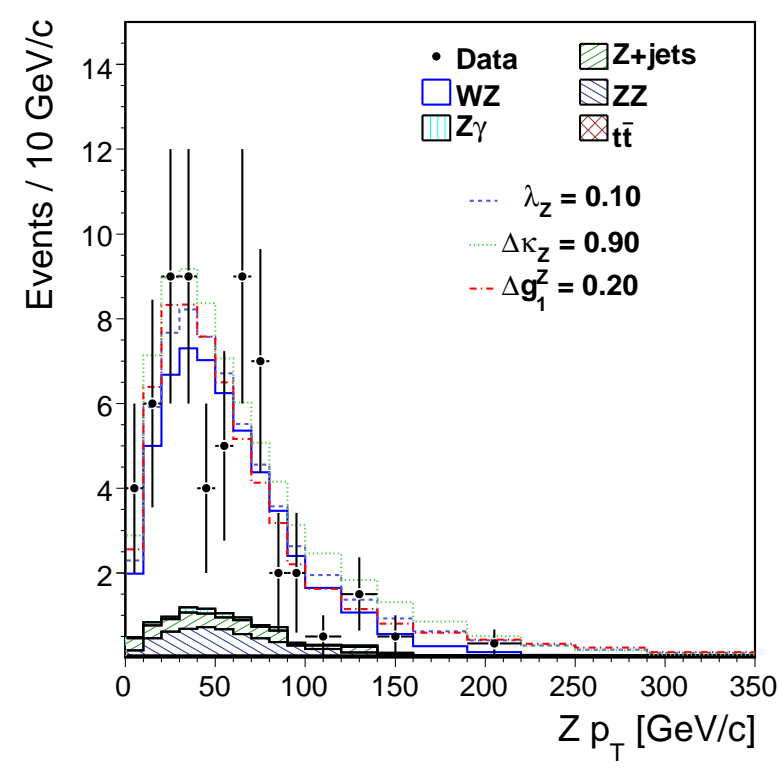

Figure 4. CDF: The $Z p_{T}$ distribution for data compared to the SM expectation for signal (WZ) and background. Also presented is how the signal expectation would change with anomalous TGCs near the observed limits.

are used to extract limit on aTGC parameters. These distributions are compared with Monte Carlo predictions for different set of coupling constants in order to evaluate $95 \%$ C.L. limits. The CDF and D $\varnothing$ collaborations are producing many competitive measurements in the diboson sector. The most updated results will be presented below.

\subsection{Anomalous TGCs limits}

The measurement of the production cross section of $W Z$ events in $p \bar{p}$ collisions has been performed by the CDF collaboration [15] with a data sample corresponding to $7.1 \mathrm{fb}^{-1}$ of integrated luminosity. The events are reconstructed in final states with three charged leptons and missing transverse energy from a neutrino. The shape and normalization of the $p_{T}$ spectrum of the $Z$ boson (see Fig. 4) are used to place $95 \%$ C.L. limits on anomalous $Z W W$ TGCs. The one-dimensional limits for a cut-off $\Lambda=2.0$ $\mathrm{TeV}$ are:

$-0.39<\Delta \kappa_{Z}<0.90$

$-0.08<\Delta \lambda_{Z}<0.10$

$-0.08<\Delta g_{1}^{Z}<0.20$

the $\mathrm{D} \emptyset$ collaboration has recently published [16] searches of the anomalous $\gamma W W$ and $Z W W$ TGCs from $W W$ and $W Z$ production using lepton plus dijet final states. A combination with results from $W \gamma$ [17], $W W$ [18] and $W Z$ [19] production with leptonic final states has been used to set the most stringent limits at hadron collider to date. The analyzed data correspond to up to $8.6 \mathrm{fb}^{-1}$.

The effects of aTGCs is to modify the high boson transverse momentum relative to its SM prediction. Therefore the corresponding $p_{T}^{j j}$ and $p_{T}^{l l}$ are used to set 95\% C.L. limits on aTGCs (see Fig 5). No deviation from SM is found
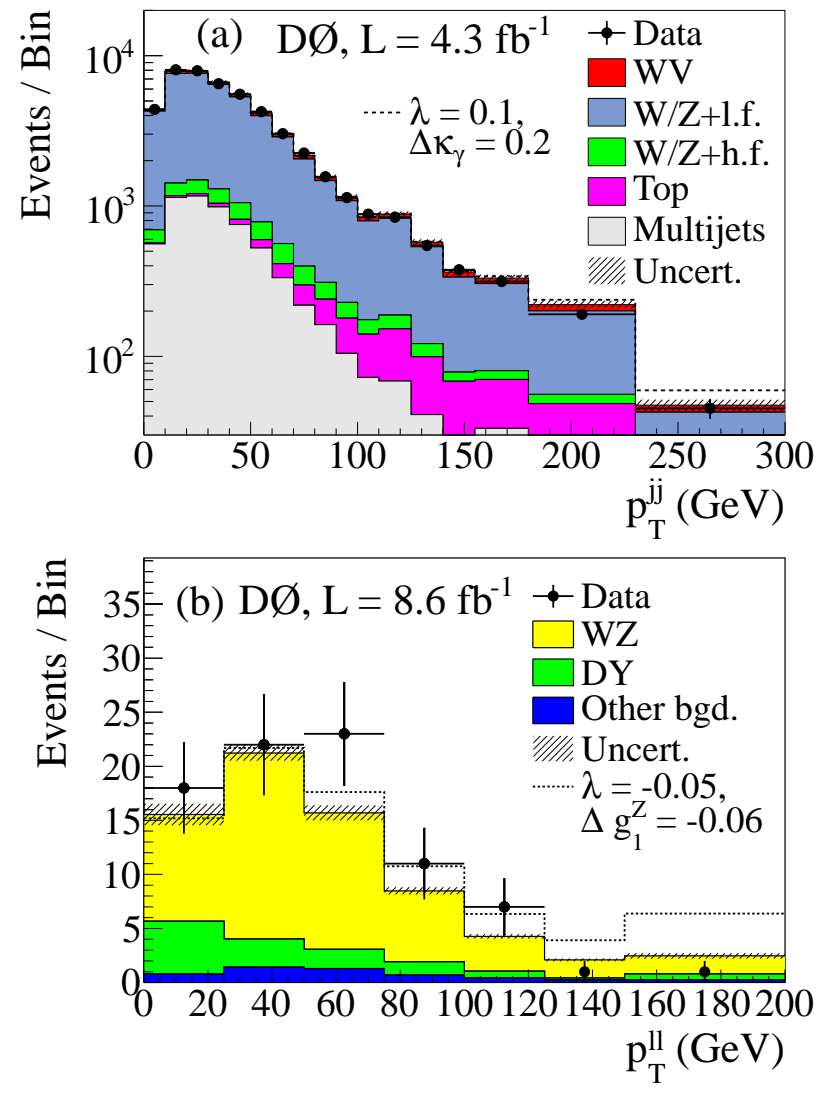

Figure 5. (a) The $p_{T}^{j j}$ distribution summed over electron and muons channels from $W W+W Z \rightarrow l v j j(l=\mu, e)$ production for data and SM MC predictions ("l.f." denotes light-partons and "h.f." denotes heavy-partons). Also shown are expected distributions for an aTGC model with $\Delta \kappa_{\gamma}=0.2$ and $\lambda=0.1$. (b) The $P_{T}^{l l}$ distribution summed over eee, $e \mu \mu$, $\mu e e$ and $\mu \mu \mu$ channels from $W Z \rightarrow l v l l$ production for data, SM MC prediction and for aTGC model with $\lambda=-0.05$ and $\Delta g_{1}^{Z}=-0.06$.

in data. The limits at 95\% C.L. from $8.6 \mathrm{fb}^{-1} \mathrm{WZ} \rightarrow l v l l$ are:

$-0.07<\lambda<0.089$ and $-0.055<\Delta g_{1}^{Z}<0.137$

while the limits from $4.3 \mathrm{fb}^{-1} \mathrm{WW}+\mathrm{WZ} \rightarrow l v j j$ are:

$-0.27<\Delta \kappa_{\gamma}<0.37$

$-0.075<\lambda<0.080$

$-0.071<\Delta g_{1}^{Z}<0.137$

Finally the overall combination gives the following $95 \%$ C.L. limits:

$-0.158<\Delta \kappa_{\gamma}<0.255$

$-0.036<\lambda<0.044$

$-0.034<\Delta g_{1}^{Z}<0.084$

Based on the combination of all diboson production and decay channels, the most stringent $68 \%$ C.L. constraints to date, are set on the $W$ boson magnetic dipole and electric quadrupole moments:

$\mu_{W}=2.012_{-0.034}^{+0.035}$ in $e / 2 M_{W}$ unit $q_{W}=-0.995_{-0.043}^{+0.042}$ in $e / M_{W}^{2}$ unit.

In beyond-the-SM scenarios, with enhanced $Z \gamma$ couplings, the photon $E_{T}$ spectrum is modified due to an enhancement in the production of high- $E_{T}$ photon [20]. The CDF collaboration has used data corresponding to $4.9 \mathrm{fb}^{-1}$ in 
the $Z \rightarrow v \bar{v}$ decay channel and $5.1 \mathrm{fb}^{-1}$ in the $Z \rightarrow l^{+} l^{-}$ $(l=\mu, e)$ decay channels [21]. With an energy cut-off of $\Lambda$ $=1.5 \mathrm{TeV}$. the $\mathrm{CP}$-conserving parameters that describe $Z \gamma$ couplings are set to be:

$\left|h_{3}^{\gamma, Z}\right|<0.022$ and $\left|h_{4}^{\gamma, Z}\right|<0.0009$

These limits are the most stringent limits to date.

The DØ Collaboration has used the transverse momentum spectrum of the photon to place limits on anomalous $Z Z \gamma$ and $Z \gamma \gamma$ couplings [22]. Data corresponding to an integrated luminosity of $6.2 \mathrm{fb}^{-1}$ in $p \bar{p} \rightarrow Z \gamma \rightarrow l^{+} l^{-} \gamma$ $(l=e, \mu)$ production has been analysed. The aTGCs limits are combined with a previous $\mathrm{D} \varnothing Z \gamma$ analysis [23] resulting in a total integrated luminosity of $7.2 \mathrm{fb}^{-1}$ in the $l l \gamma$ decay channel and $3.6 \mathrm{fb}^{-1}$ in the $v v \gamma$ decay channel. Applying an energy cut-off of $\Lambda=1.5 \mathrm{TeV}$ the limits at $95 \%$ C.L. are set to be:

$\left|h_{3}^{Z}\right|<0.026$ and $\left|h_{4}^{Z}\right|<0.0013$

$\left|h_{3}^{\gamma}\right|<0.027$ and $\left|h_{4}^{\gamma}\right|<0.0014$

These limits are comparable to those found in the most recent CDF result reported above.

\subsection{Conclusion}

A new precision measurement of the $W$ boson mass performed by $\mathrm{CDF}$ and $\mathrm{D} \varnothing$ Collaborations has improved the precision of the Tevatron average to $16 \mathrm{MeV}$, which combined with the LEP average slightly reduces the new world average to $15 \mathrm{MeV}$. These improvments in the experimental precision on $M_{W}$ lead to precise indirect constraints on the mass of the Higgs boson. The direct measurements of the mass of the Higgs boson candidate discovered at the LHC agree, at $1.2 \sigma$ level, with indirect constraints. Even if this is a remarkable success for the standard model, more precise measurements of $M_{W}$ are needed to tighten the indirect constraints and make this test of SM even more stringent. The precision of both $\mathrm{CDF}$ and $\mathrm{D} \emptyset$ experiments is expected to improve significantly once the full data set (almost $10 \mathrm{fb}^{-1}$ ) has been analysed.

The CDF and D $\varnothing$ Collaborations have presented recent results of diboson production using the data collected at the Tevatron between 2002-2011. The limits on anomalous TGCs parameters are competitive with previous results from the LEP and first results from the LHC. They are consistent with the SM expectations. CDF and DØ can anticipate 2 to 10 times more data (depending on the channels), to cope with the full integrated luminosity $\left(10 \mathrm{fb}^{-1}\right)$ at Tevatron. This will result in an increase of the aTGCs sensitivities by a factor 3 to 5 after a combination of the $\mathrm{CDF}$ and $\mathrm{D} \emptyset$ results.

\section{References}

[1] C. Balazs, and C.P. Yuan, Phys. Rev. D56 (1997) 5558.
[2] E. Barberio, and Z. Was, Comput. Phys. Commun. 79 291 (1994)

[3] P.M. Nadolsky, H.L. Lai, Q.H. Cao, J. Huston, J. Pumplin, D. Stump, W.K. Tung, and C.P. Yuan, Phys. Rev. D78, 3004 (2008).

[4] F. Landry, R. Brocks, P.Nadolsky, and C.P. Yuan, Phys. Rev. D67, 073016 (2003).

[5] T.Aaltonen et al., The CDF Collaboration, Phys. Rev. Lett. 108, 151803 (2012).

[6] V.M. Abazov et al., The D $\varnothing$ Collaboration, Phys. Rev. Lett. 108, 151804 (2012).

[7] ALEPH Collaboration, DELPHI Collaboration, L3 Collaboration, OPAL Collaboration, LEP Electroweak Working Group, and SLD Electroweak and Heavy Flavor Groups, Phys. Rept. B 427, 257 (2006).

[8] A. Valassi, Nucl. Instrum. Methods Phys. Res., Sect A500, 391 (2003).

[9] V.M. Abazov et al. (DØ Collaboration), Phys. Rev. Lett. 103, 141801 (2009).

[10] The Tevatron electroweak working group for the CDF and D $\varnothing$ collaborations, FERMILAB-TM-2532-E, arXiv:1204.0042 (2012).

[11] M. Baak et al. (Gfitter Group), Eur. Phys. J. C72 2205 (2012).

[12] T. Aaltonen et al., (CDF and DØ Collaborations), Phys. Rev. D86, 092003 (2012).

[13] K. Hagiwara, J. Woodside and D. Zeppenfeld, Phys. Rev. D41, 21113 (1990).

[14] C. Amsler et al. (Particle Data Group), Phys. Lett. B667, (2008) 1.

[15] T. Aaltonen et al., The CDF Collaboration, Phys. Rev. D86, 031104 (2012).

[16] V.M. Abazov et al., The DØ Collaboration, Phys. Lett. B718, 451 (2012).

[17] V.M. Abazov et al., The DØ Collaboration, Phys. Rev. Lett. 107, 241803 (2011).

[18] V.M. Abazov et al., The DØ Collaboration, Phys. Rev. Lett. 103, 191801 (2009).

[19] V.M. Abazov et al., The DØ Collaboration, Phys. Rev. D80, 053012 (2009).

[20] U. Baur and E.L. Berger, Phys. Rev. D47, 4889 (1993)

[21] T. Aaltonen et al., The CDF Collaboration, Phys. Rev. Lett. 107, 051802 (2011).

[22] V.M. Abazov et al., The DØ Collaboration, Phys. Rev. D85, 052001 (2012).

[23] V.M. Abazov et al. The DØ Collaboration, Phys. Rev. Lett. 102, 201802 (2009). 